\title{
Modeling Process of Hot Isostatic Pressing with Nonstationary Temperature Field
}

\author{
Bochkov A.V., Kozyrev Y.M., Ponomarev A.V.* \\ Moscow State University of Instrument Making and Computer Science, Moscow, Russia \\ *Corresponding Author: avpon@yandex.ru
}

Copyright (C) 2014 Horizon Research Publishing All rights reserved.

\begin{abstract}
In the article we consider the problems of modern powder metallurgy. In particular, we describe the method of modeling powder material dynamics during hot isostatic pressing. The modeling results are compared with experimental data.
\end{abstract}

Keywords HIP Process, Powder Material, Non-Uniform Temperature Field, Yield Criterion of Green

\section{Introduction}

These days more materials and structures with improved performance characteristics are needed. Powder metallurgy is one of the ways to obtain these kinds of structures by means of using powder material as raw product.

One of the methods of powder metallurgy is hot isostatic pressing (HIP) which is based on compaction of powder material under high pressure (about 1000 atm.) and high temperatures (about $1000^{\circ} \mathrm{C}$ ). The word "isostatic" is explained by historical reasons and is not related to the actual physics of the process. In a capsule with certain initial geometry the powder material and embedded elements (forming the cavity in the final product) are placed. Then the air is removed from the capsule and it is placed in gasostat, where under the influence of gas (argon), the process of the HIP takes place. In the last step the capsule and embedded elements are removed (chemically or mechanically) to form a final product.

The main problem of HIP is to obtain the desired geometry of product with a sufficient degree of accuracy. Often, the customer has to pay for several experimental samples of the product. This is extremely disadvantageous in small-scale production, considering the high cost of raw materials and processing.

There are two main reasons that cause this problem. First reason is the difference of mechanical properties of the capsule, a powder material and embedded elements. The second reason is temperature non-uniformity of powder material during the HIP process. Temperature non-uniformity is a consequence of high degree of vacuum inside the capsule and as a result, the heating of powder material is extremely slow and, also, it is technically impossible to measure the temperature distribution over the whole product. The consequences of the described problems are discussed in [1].

To address all of these issues the software package was developed by "Laboratory of New Technologies" (Moscow), in collaboration with the Moscow State University of Instrument Making and Computer Science.

The developed software package enables to simulate HIP processes and predict with high accuracy the geometry of the final product.

It was shown in [2] that during the modeling of HIP process the powder material can be regarded as a solid medium. The calculation is performed by finite element method taking into account the effect of plastic deformation. Elastic deformation and the viscosity not taken into account because of their insignificance in the process.

Most products obtained by the HIP have an axisymmetric geometry; therefore the initial geometry of the capsule is defined as half of the longitudinal section (along the symmetrical axis).

First, we manually split the drawing into the finite elements, then initial temperature and density are chosen, and the database of materials types is used to classify the finite elements. For each material type in database the following experimental based data is available: yield strength, thermal conductivity, heat capacity and bulk density as a function of temperature and functions $f_{1}, f_{2}$ of material density $\rho$. Also the dynamics of the external temperature and pressure is selected manually.

\section{Method of Calculation}

The core feature of HIP process modeling method is an iterative scheme for calculating the Lame parameters: $\lambda$ and $\mu$.

First, let's introduce the general mathematical formulation of HIP modeling problem.

1. The equilibrium equation:

$$
\operatorname{div} \bar{\sigma}=0
$$


where $\bar{\sigma}-$ the stress tensor.

2. The equation of the yield surface:

$$
\Phi\left(\sigma_{i j}, \rho, T\right)=0
$$

where $\rho$-relative density, $T$ - temperature.

3. The dependence of the stress tensor from the strain rate tensor is defined by the associated flow law:

$$
\dot{\varepsilon}_{i j}=\beta \frac{\partial \Phi}{\partial \sigma_{i j}}
$$

4. To describe the behavior of the capsule material and the embedded element the condition of perfect plasticity is assumed:

$$
S^{2}=\sigma_{S}^{2}
$$

where $\sigma_{S}$ - is the yield limit, $S^{2}-$ the second invariant of the stress tensor deviator.

5. The condition of incompressibility:

$$
\operatorname{div} \bar{u}=0
$$

where $\bar{u}$ - movement velocity.

6. To calculate the density the equation of continuity is used.

$$
\frac{d \rho}{d t}+\rho \operatorname{div} \bar{u}=0
$$

where $\bar{u}$ - movement velocity.

7. To determine the temperature the heat equation is used:

$$
\frac{\partial T}{\partial t}=\operatorname{div} \lambda(\rho) \operatorname{grad} T
$$

The problem is considered in a quasi-static formulation, the deformation process is considered to be slow, therefore the acceleration in the equations of equilibrium is neglected. Thermal expansion of the material is also neglected. At the boundary between "powder - capsule" and "powder embedded element" continuity condition of the displacement field is supposed.

It should be noted that the thermal conductivity of the powder material varies by about 2 orders of magnitude under the condition of relative density dynamics during HIP process. With the temperature changes from $20 \mathrm{C}$ to $1120 \mathrm{C}$, yield strength varies by order of magnitude. The behavior of the powder material in a non-uniform transient temperature field was analyzed in [3].

To describe the mechanical properties of the powder material yield criterion of Green is used [4-5]:

$$
\frac{\sigma^{2}}{f_{2}^{2}}+\frac{S^{2}}{f_{1}^{2}}=\sigma_{S}^{2}
$$

$\sigma=\sigma_{i i} / 3-$ the first invariant of the stress tensor; $\sigma_{S}-$ is the yield limit of the powder material after complete densification $(\rho=1)$, that is a known function of temperature;

$$
S^{2}=\frac{3}{2}\left(\sigma_{i j}-\sigma \delta_{i j}\right)\left(\sigma_{i j}-\sigma \delta_{i j}\right) .
$$

The equation of the yield surface can be written as:

$$
\begin{gathered}
\frac{\left(\sigma_{r}+\sigma_{\varphi}+\sigma_{z}\right)^{2}}{9 f_{2}^{2}}+\frac{1}{6 f_{1}^{2}}\left[\left(2 \sigma_{r}-\sigma_{\varphi}-\sigma_{z}\right)^{2}+\right. \\
\left.+\left(2 \sigma_{\varphi}-\sigma_{r}-\sigma_{z}\right)^{2}+\left(2 \sigma_{z}-\sigma_{r}-\sigma_{\varphi}\right)^{2}+18 \sigma_{r z}^{2}\right]=\sigma_{S}^{2}
\end{gathered}
$$

According to associated flow law we can write the following:

$$
\begin{aligned}
& \varepsilon_{r P}=\beta\left[\frac{2\left(\sigma_{r}+\sigma_{\varphi}+\sigma_{z}\right)}{9 f_{2}^{2}}+\frac{2 \sigma_{r}-\sigma_{\varphi}-\sigma_{z}}{f_{1}^{2}}\right] \\
& \varepsilon_{\varphi P}=\beta\left[\frac{2\left(\sigma_{r}+\sigma_{\varphi}+\sigma_{z}\right)}{9 f_{2}^{2}}+\frac{2 \sigma_{\varphi}-\sigma_{r}-\sigma_{z}}{f_{1}^{2}}\right] \\
& \varepsilon_{z P}=\beta\left[\frac{2\left(\sigma_{r}+\sigma_{\varphi}+\sigma_{z}\right)}{9 f_{2}^{2}}+\frac{2 \sigma_{z}-\sigma_{r}-\sigma_{\varphi}}{f_{1}^{2}}\right] \\
& \varepsilon_{r z P}=\beta \frac{3}{f_{1}^{2}} \sigma_{r z}
\end{aligned}
$$

where $\varepsilon_{r P}, \varepsilon_{\varphi P}, \varepsilon_{z} P, \varepsilon_{r z} P$ - rates of plastic deformation.

Solving for $\sigma_{r}, \sigma_{\varphi}, \sigma_{z}$ and using yield criterion of Green, we derive the following:

$$
\begin{gathered}
\frac{1}{\beta^{2}}\left\{\frac{f_{2}^{2}}{4}\left(\varepsilon_{r} P+\varepsilon_{\varphi P}+\varepsilon_{z} P\right)^{2}+\frac{f_{1}^{2}}{54} \times\right. \\
3 \times\left[\left(2 \varepsilon_{r} P-\varepsilon_{\varphi P}-\varepsilon_{z P}\right)^{2}+\left(2 \varepsilon_{\varphi P}-\varepsilon_{r} P-\varepsilon_{z} P\right)^{2}+\right. \\
\left.\left.+\left(2 \varepsilon_{z} P-\varepsilon_{r} P-\varepsilon_{\varphi P}\right)^{2}+18 \varepsilon_{r z}^{2}\right]\right\}=\sigma_{S}^{2}
\end{gathered}
$$

Let us introduce the notation:

$$
\begin{gathered}
\theta_{P}=\varepsilon_{r} P+\varepsilon_{\varphi P}+\varepsilon_{z P} \\
H_{P}^{2}=\frac{2}{27}\left[\left(2 \varepsilon_{r P}-\varepsilon_{\varphi P}-\varepsilon_{z P}\right)^{2}+\left(2 \varepsilon_{\varphi P}-\varepsilon_{r P}-\varepsilon_{z P}\right)^{2}+\right. \\
\left.+\left(2 \varepsilon_{z P}-\varepsilon_{r P}-\varepsilon_{\varphi P}\right)^{2}+18 \varepsilon_{r z P}^{2}\right]
\end{gathered}
$$

then the equation of the yield surface will have the following form:

$$
\frac{1}{\beta^{2}}\left\{\frac{1}{4} f_{2}^{2} \theta_{P}^{2}+\frac{1}{4} f_{1}^{2} H_{P}^{2}\right\}=\sigma_{S}^{2}
$$

and the stress tensor can be expressed as:

$$
\begin{gathered}
\sigma_{r}=\frac{2 \sigma_{S}}{\sqrt{f_{2}^{2} \theta_{P}^{2}+f_{1}^{2} H_{P}^{2}}}\left[\frac{9 f_{2}^{2}+4 f_{1}^{2}}{18} \varepsilon_{r P}+\right. \\
\left.+\frac{9 f_{2}^{2}-2 f_{1}^{2}}{18} \varepsilon_{\varphi P}+\frac{9 f_{2}^{2}-2 f_{1}^{2}}{18} \varepsilon_{z P}\right]
\end{gathered}
$$




$$
\begin{aligned}
& \sigma_{\varphi}=\frac{2 \sigma_{S}}{\sqrt{f_{2}^{2} \theta_{P}^{2}+f_{1}^{2} H_{P}^{2}}}\left[\frac{9 f_{2}^{2}-2 f_{1}^{2}}{18} \varepsilon_{r P}+\right. \\
& \left.+\frac{9 f_{2}^{2}+4 f_{1}^{2}}{18} \varepsilon_{\varphi P}+\frac{9 f_{2}^{2}-2 f_{1}^{2}}{18} \varepsilon_{z P}\right] \\
& \sigma_{z}=\frac{2 \sigma_{S}}{\sqrt{f_{2}^{2} \theta_{P}^{2}+f_{1}^{2} H_{P}^{2}}}\left[\frac{9 f_{2}^{2}-2 f_{1}^{2}}{18} \varepsilon_{r P}+\right. \\
& \left.+\frac{9 f_{2}^{2}-2 f_{1}^{2}}{18} \varepsilon_{\varphi P}+\frac{9 f_{2}^{2}+4 f_{1}^{2}}{18} \varepsilon_{z} P\right] \\
& \sigma_{r z}=\frac{2 \sigma_{S}}{\sqrt{f_{2}^{2} \theta_{P}^{2}+f_{1}^{2} H_{P}^{2}}} \frac{f_{1}^{2}}{3} \varepsilon_{r z P}
\end{aligned}
$$

The relationship between stress tensor and rate of deformation, according to Hooke's law takes the form:

$$
\begin{aligned}
& \sigma_{r}=\lambda\left(\varepsilon_{r}+\varepsilon_{\varphi}+\varepsilon_{z}\right)+2 \mu \varepsilon_{r} \\
& \sigma_{\varphi}=\lambda\left(\varepsilon_{r}+\varepsilon_{\varphi}+\varepsilon_{z}\right)+2 \mu \varepsilon_{\varphi} \\
& \sigma_{z}=\lambda\left(\varepsilon_{r}+\varepsilon_{\varphi}+\varepsilon_{z}\right)+2 \mu \varepsilon_{z} \\
& \sigma_{r z}=2 \mu \varepsilon_{r z}
\end{aligned}
$$

For an elastic case, solving for the rate of deformation, we derive:

$$
\begin{aligned}
& \varepsilon_{r E}=\frac{\lambda+\mu}{\mu(3 \lambda+2 \mu)} \sigma_{r}-\frac{\lambda}{2 \mu(3 \lambda+2 \mu)} \sigma_{\varphi}- \\
& -\frac{\lambda}{2 \mu(3 \lambda+2 \mu)} \sigma_{z} \\
& \varepsilon_{\varphi E}=-\frac{\lambda}{2 \mu(3 \lambda+2 \mu)} \sigma_{r}+\frac{\lambda+\mu}{\mu(3 \lambda+2 \mu)} \sigma_{\varphi}- \\
& -\frac{\lambda}{2 \mu(3 \lambda+2 \mu)} \sigma_{z} \\
& \varepsilon_{z E}=-\frac{\lambda}{2 \mu(3 \lambda+2 \mu)} \sigma_{r}-\frac{\lambda}{2 \mu(3 \lambda+2 \mu)} \sigma_{\varphi}+ \\
& +\frac{\lambda+\mu}{\mu(3 \lambda+2 \mu)} \sigma_{z} \\
& \varepsilon_{r z E}=\frac{1}{2 \mu} \sigma_{r z}
\end{aligned}
$$

Hence, the Young's modulus and Poisson's ratio may be expressed as:

$$
E=\frac{\mu(3 \lambda+2 \mu)}{\lambda+\mu} ; \quad v=\frac{\lambda}{2(\lambda+\mu)}
$$

and Lame parameters take the form:

$$
\begin{gathered}
\mu=\frac{E}{2(1+v)} \\
\lambda=\frac{E v}{(1+v)(1-2 v)}=\frac{E}{3(1-2 v)}-\frac{2}{3} \mu
\end{gathered}
$$

Then the expressions for the elastic deformation will be:

$$
\begin{aligned}
& \varepsilon_{r E}=\frac{1}{E} \sigma_{r}-\frac{v}{E} \sigma_{\varphi}-\frac{v}{E} \sigma_{z} \\
& \varepsilon_{\varphi E}=-\frac{v}{E} \sigma_{r}+\frac{1}{E} \sigma_{\varphi}-\frac{v}{E} \sigma_{z} \\
& \varepsilon_{z E}=-\frac{v}{E} \sigma_{r}-\frac{v}{E} \sigma_{\varphi}+\frac{1}{E} \sigma_{z} \\
& \varepsilon_{r z E}=\frac{1+v}{E} \sigma_{r z}
\end{aligned}
$$

Then from (19), we derive:

$$
\begin{aligned}
& \sigma_{r}+\sigma_{\varphi}+\sigma_{z}= \\
& =\frac{3 \frac{E}{1-2 v} \sigma_{S} f_{2}^{2}}{\frac{E}{1-2 v} \sqrt{f_{2}^{2} \theta_{P}^{2}+f_{1}^{2} H_{P}^{2}}+3 \sigma_{S} f_{2}^{2}}\left(\varepsilon_{r}+\varepsilon_{\varphi}+\varepsilon_{z}\right) \\
& \sigma_{r z}=\frac{2 \mu \sigma_{S} \frac{f_{1}^{2}}{3}}{\mu \sqrt{f_{2}^{2} \theta_{P}^{2}+f_{1}^{2} H_{P}^{2}}+\sigma_{S} \frac{f_{1}^{2}}{3}} \varepsilon_{r z}
\end{aligned}
$$

where $\varepsilon_{r}, \varepsilon_{\varphi}, \varepsilon_{z}, \varepsilon_{r z} \quad-$ rates of elastic-plastic deformation.

Finally, the expression for the elastic moduli takes the form:

$$
\begin{gathered}
\tilde{\mu}=\frac{\mu \sigma_{S} \frac{f_{1}^{2}}{3}}{\mu \sqrt{f_{2}^{2} \theta_{P}^{2}+f_{1}^{2} H_{P}^{2}}+\sigma_{S} \frac{f_{1}^{2}}{3}} \\
\tilde{\lambda}=\frac{\frac{E}{1-2 v} \sigma_{S} f_{2}^{2}}{\frac{E}{1-2 v} \sqrt{f_{2}^{2} \theta_{P}^{2}+f_{1}^{2} H_{P}^{2}}+3 \sigma_{S} f_{2}^{2}}-\frac{2}{3} \tilde{\mu}
\end{gathered}
$$

The calculated values of the Lame parameters are used in the modeling of HIP process.

\section{Modeling Procedure}

The modeling procedure is based on estimation of product state at certain time intervals. The time step in seconds is a parameter of the algorithm.

At the beginning of each step deformations are considered to be purely elastic and calculated according to formulas (2). Next, there is an iterative process of calculating the deformations. On each step of the algorithm the following convergence condition is checked: deformations differences 
between successive steps of algorithm cannot exceed initially given threshold value.

If the convergence condition is not satisfied, the value of Lame parameters is recalculated in the following way: if the condition of $\frac{\sigma^{2}}{f_{2}^{2}}+\frac{S^{2}}{f_{1}^{2}}<\sigma_{S}^{2}$ is satisfied, an element is considered to be elastic and formulas (20) are used. If the condition above is not satisfied then the element is exposed to plastic deformation and Lame parameters are calculated by the formulas (22).

If the convergence condition is satisfied, the final calculation is carried out with the latest values of the Lame parameters.

It should be noted that the convergence of the method was not proved theoretically, but the algorithm is very effective on practice. When plastic deformations exist in powder material, on average, it takes only 20 steps for algorithm to converge.

Analysis of non-uniform temperature field using the above algorithm has been made in [6-7].

\section{Modeling Example}

As an example, let's take a powder tube (without a capsule and embedded elements, so there are no actual experiment provided for this cases), with inner radius $\mathrm{R} 1=1000 \mathrm{~mm}$ and an external $\mathrm{R} 2=1400 \mathrm{~mm}$, length $\mathrm{H}=1600 \mathrm{~m}$. Figure 1 below shows half of its longitudinal section.

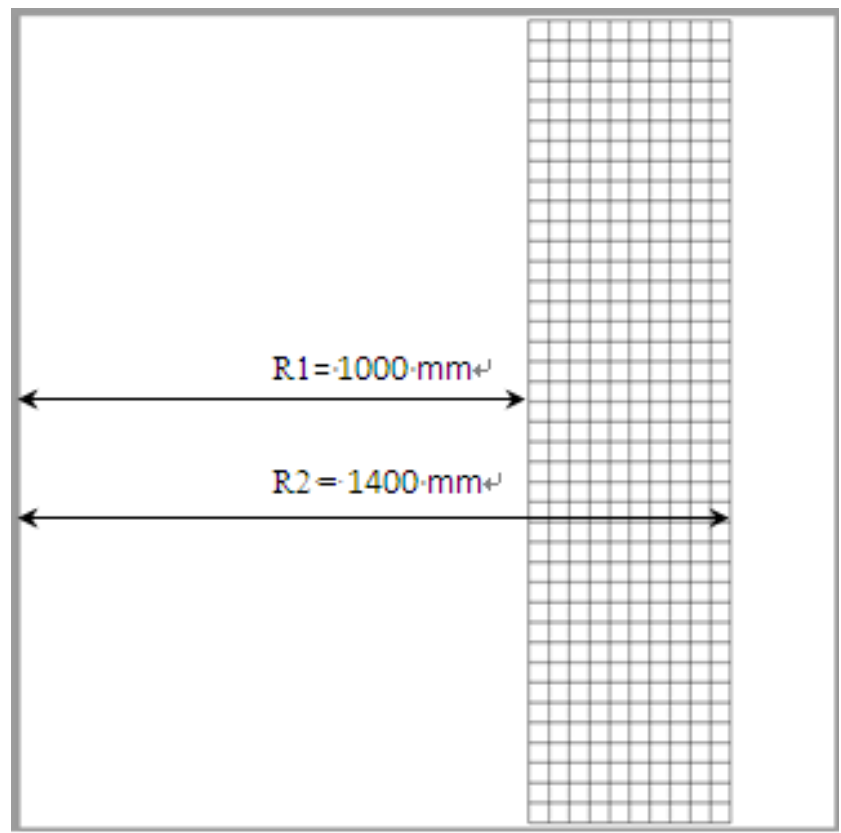

Figure 1. Initial geometry

In order to find out how different temperature conditions influence on the geometry of the final product we made two simulations (without actual experiment provided because capsule not used):
1) At constant temperature $\left(1121^{\circ} \mathrm{C}\right.$ ) (for HIP cycle see table 1)

Initial powder material (Astroloy) density was $68 \%$.

2) At ranging temperature (from $20^{\circ} \mathrm{C}$ to $1121^{\circ} \mathrm{C}$, for HIP cycle see table 2)

Table 1. Simulation 1. HIP Cycle

\begin{tabular}{|c|c|c|}
\hline Time $(\mathrm{sec})$ & Temperature $(\mathrm{C})$ & Pressure (MPa) \\
\hline 0 & 1121 & $0-30$ \\
\hline 2700 & 1121 & $30-47$ \\
\hline 5400 & 1121 & $47-65$ \\
\hline 8100 & 1121 & $65-82$ \\
\hline 10800 & 1121 & $82-101$ \\
\hline
\end{tabular}

Table 2. Simulation 2. HIP Cycle

\begin{tabular}{|c|c|c|}
\hline Time $(\mathrm{sec})$ & Temperature $(\mathrm{C})$ & Pressure (MPa) \\
\hline 0 & 20 & $0-30$ \\
\hline 2700 & 300 & $30-47$ \\
\hline 5400 & 600 & $47-65$ \\
\hline 8100 & 900 & $65-82$ \\
\hline 10800 & 1121 & $82-101$ \\
\hline
\end{tabular}

Initial powder material (Astroloy) density was $68 \%$.

Figure 2 shows the final product geometry, calculated at constant temperature condition. As can be seen from the figure, the product has dimensions: $\mathrm{R} 1=880 \mathrm{~mm}, \mathrm{R} 2=1232$ $\mathrm{mm}, \mathrm{H}=1408 \mathrm{~mm}$.

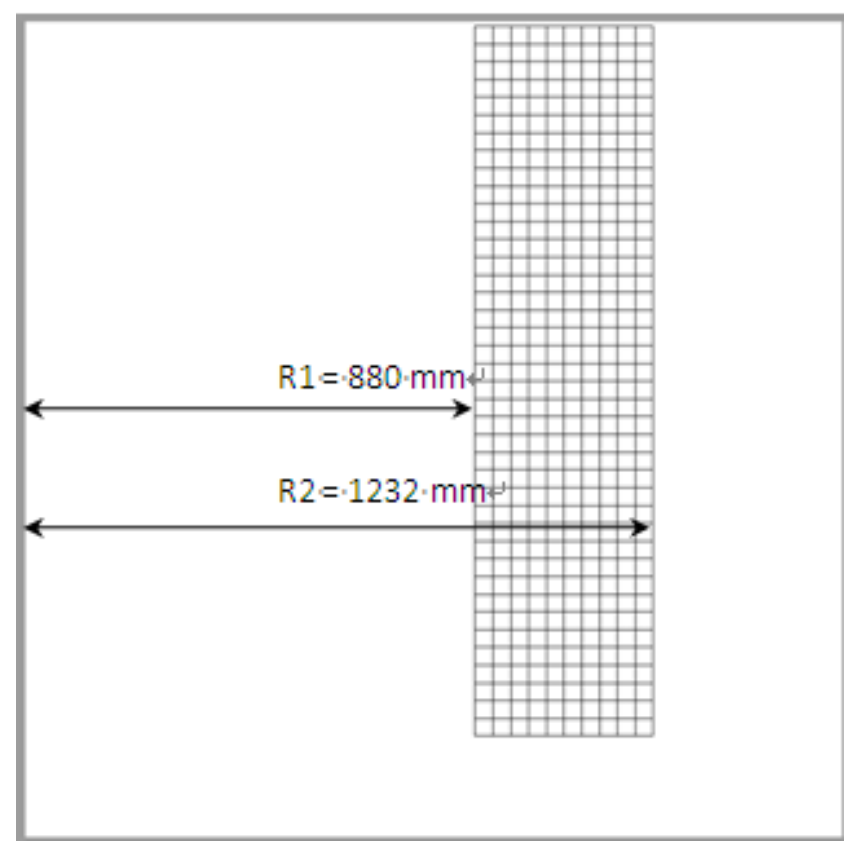

Figure 2. Estimated geometry under a constant temperature condition $1121 \mathrm{C}$

Figure 3 shows the final product geometry, calculated at a 
variable temperature. As can be seen from the figure, the geometry of the product is significantly distorted $\mathrm{R} 1=930$ $\mathrm{mm}, \mathrm{R} 2=1264 \mathrm{~mm}, \mathrm{R} 3=934 \mathrm{~mm}, \mathrm{R} 4=1280 \mathrm{~mm}, \mathrm{H}=$ $1430 \mathrm{~mm}$.

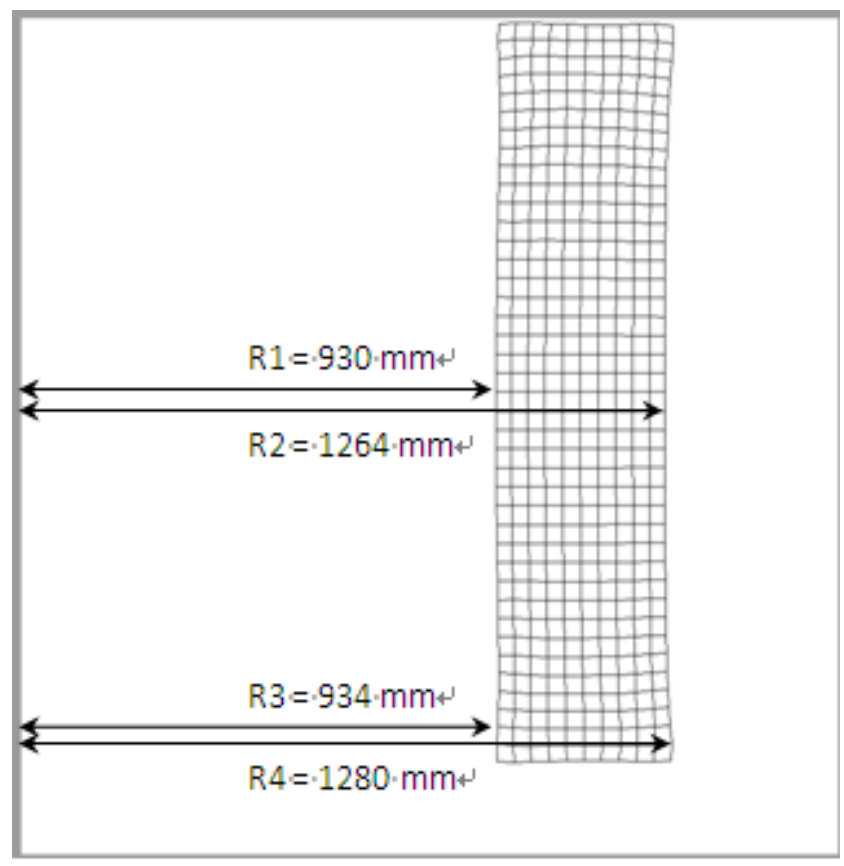

Figure 3. Estimated geometry under variable temperature condition $20 \mathrm{C}$ $-1121 \mathrm{C}$

The modeling results suggest that the final product geometry is strongly influenced by the temperature conditions.

We also give an example of the calculation of the actual product (Figure 4) and the results of the pilot production (Figure 5) at raging temperature and pressure (for HIP cycle see table 3).

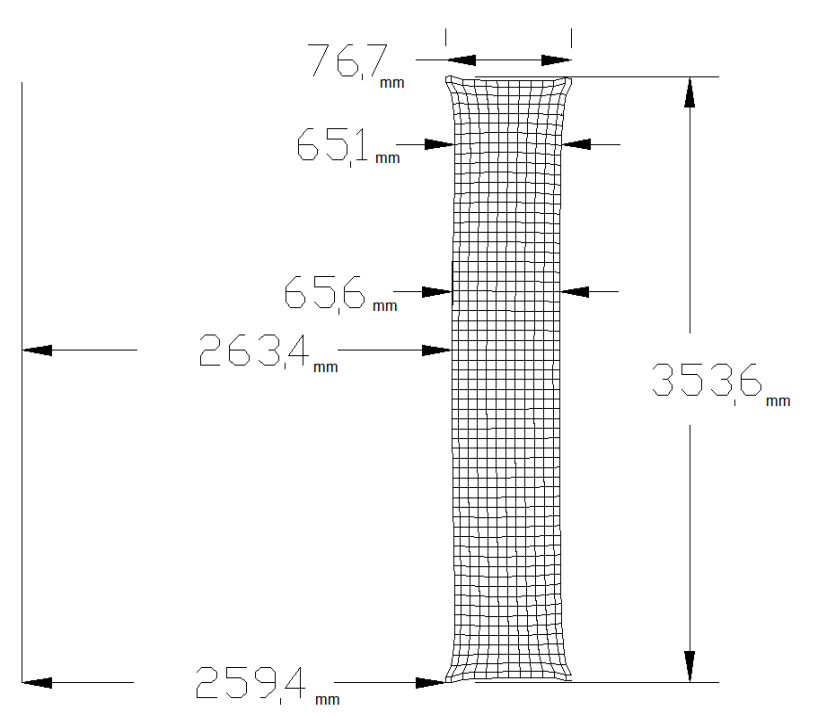

Figure 4. Estimated actual geometry

HIP cycle was (boundary conditions):
Table 3. Simulation 3. HIP Cycle

\begin{tabular}{|c|c|c|}
\hline Time $(\mathrm{sec})$ & Temperature $(\mathrm{C})$ & Pressure $(\mathrm{MPa})$ \\
\hline 0 & 20 & $0-30$ \\
\hline 2700 & 300 & $30-47$ \\
\hline 5400 & 600 & $47-65$ \\
\hline 8100 & 900 & $65-82$ \\
\hline 10800 & 1121 & $82-101$ \\
\hline
\end{tabular}

Initial powder material (Astroloy) density was $68 \%$ and A1018 for capsule material.

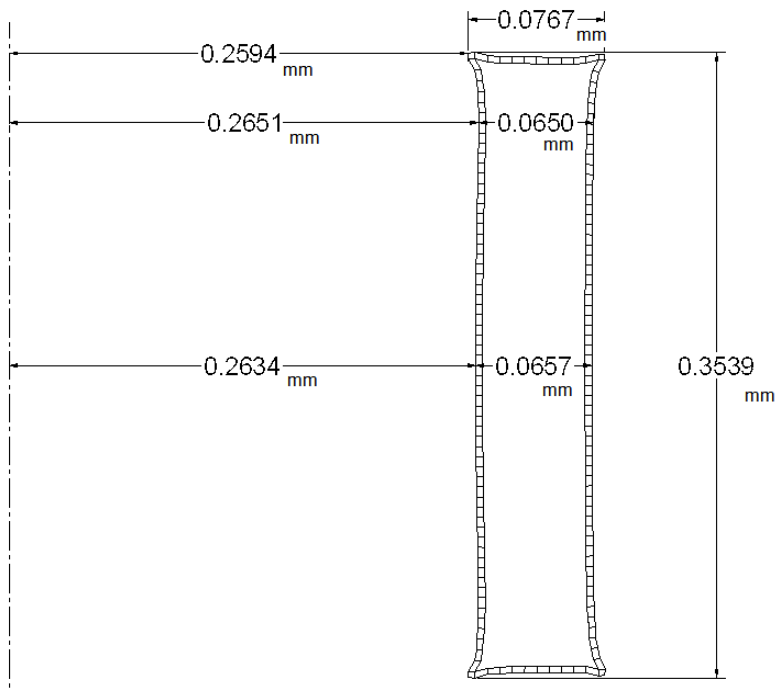

Figure 5. Actual geometry

\section{Conclusion}

The proposed method can achieve considerable accuracy of HIP process modeling (average error less than $0.8 \%-$ $1.2 \%$ ), and was successfully tested on the products of complex geometry (LNT, Russia).

\section{REFERENCES}

[1] Ponomarev A.V. Mathematical modeling of powder material deformations. - LAMBERT Academic Publishing, 2011.

[2] Fedorenko I.M., Andrievsky V.A. Fundamentals of powder metallurgy. - Kiev, publ. AS USSR, 1963.

[3] Druyanov B.A., Samarov V.N. Compaction of the powder material in a non-uniform temperature field. "Powder metallurgy », 1989, \#3.

[4] Druyanov B.A. Applied theory of plasticity of porous bodies. - M.: Mashinostroyeniye, 1989.

[5] Green G. The theory of porous bodies plasticity. - the first collection of translations. «Mechanics», 1973, \#4, p. 109-120. 
[6] Bochkov A.V., Goloveshkin V.A., Pirumov A.R., Ponomarev A.V., Samarov V.N. The influence of inhomogeneous non-stationary temperature field on the HIP process of pipes. Fundamental and applied problems of engineering and technology. Scientific - technical journal Orel GTU, \# 6 (284), 2010, P. 3-9.
[7] Bochkov A.V., Goloveshkin V.A., Kozyrev Y.M., Ponomarev A.V., Samarov V.N. The compaction process of powder materials in a non-uniform transient temperature field. Mechanics of composite materials and structures. - T.11. -\#3. -2011 - p. 401-410. 